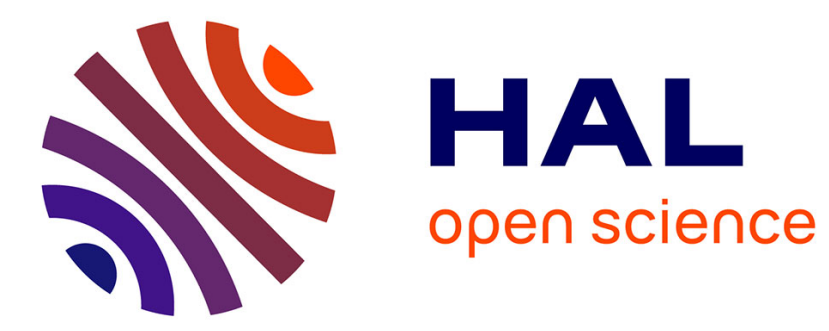

\title{
Alkali metal atomic transition probability in strong external magnetic field and its application
}

Claude Leroy, G. Hakhumyan, Y. Pashayan-Leroy, D. Sarkisyan

\section{To cite this version:}

Claude Leroy, G. Hakhumyan, Y. Pashayan-Leroy, D. Sarkisyan. Alkali metal atomic transition probability in strong external magnetic field and its application. International Conference on Laser Physics 2010, Oct 2010, Ashtarak, Armenia. pp.79980S, 10.1117/12.890915 . hal-00620515

\section{HAL Id: hal-00620515 https://hal.science/hal-00620515}

Submitted on 7 Sep 2011

HAL is a multi-disciplinary open access archive for the deposit and dissemination of scientific research documents, whether they are published or not. The documents may come from teaching and research institutions in France or abroad, or from public or private research centers.
L'archive ouverte pluridisciplinaire HAL, est destinée au dépôt et à la diffusion de documents scientifiques de niveau recherche, publiés ou non, émanant des établissements d'enseignement et de recherche français ou étrangers, des laboratoires publics ou privés. 


\title{
Alkali metal atomic transition probability in strong external magnetic field and its application
}

\author{
C. Leroy $^{1}$, G. Hakhumyan ${ }^{1,2}$, Y. Pashayan-Leroy ${ }^{1}$, D. Sarkisyan ${ }^{2}$ \\ ${ }^{1}$ Laboratoire Interdisciplinaire Carnot de Bourgogne, UMR CNRS 5209 - Université de \\ Bourgogne, F-21078 Dijon Cedex, France \\ ${ }^{2}$ Institute for Physical Research, NAS of Armenia, Ashtarak, 0203, Armenia
}

\begin{abstract}
Interaction of alkali atoms with external magnetic field induced a splitting and a shift of their energy levels. We have study this interaction for external field from 0 to 5000 Gauss when the alkali vapor is confined in submicron thin vapor cell with thickness $L=\lambda / 2$. Rubidium and Sodium vapors have been studied. The Hamiltonian can be expressed as the sum of the unperturbated atomic Hamiltonian and the so-called Zeeman Hamiltonian. The probability of a transition, induced by the laser electric field is proportional to the square of the transfer coefficients modified by the presence of the magnetic field. We will show that the strong nonlinearity of the transition intensities versus the external magnetic field intensity is obvious for $B>100 \mathrm{G}$. Some possible application for Laser Spectroscopy of the $\mathrm{Rb}$ and the $\mathrm{Na}$ atoms are presented
\end{abstract}

Keywords: Zeeman Hamiltonian, atomic transition intensity, frequency shift, submicron thin vapor.

\section{INTRODUCTION}

Optical nano-cells (NCs) containing atomic vapor of alkali metal have already proved their efficiency to study atomic phenomena with a precision that normal size cells cannot achieve. One could even define as elegant tool such a device when used for the interpretation of interaction between atom and light, atom and surface or atom and atom $^{1-8}$.

A NC has one of his dimensions $L$ of nanometric thickness, that is, $L$ can vary continuously from 0.1 to $10 \lambda$ where $\lambda$ is the laser wavelength resonant with the considered atomic transition while the two others dimensions are of centimeter order.

Furthermore, one of the main advantages of this elegant tool appears when one considers the atomic transitions of alkali atoms in magnetic fields. Indeed, for centimeter size thickness cell, the splitting of these transitions into their Zeeman components is relatively bad resolved due mainly to Doppler broadening and the resolution becomes even worst when the external magnetic field increases: well-known saturation absorption method allows only studying the behaviour of a total set of hyperfine transitions and becomes useless for magnetic field such that $B>100 \mathrm{G}$ if one wants to describe individual hyperfine transition.

The high resolution atomic spectra obtained with NCs come from the possibility to highly reduce the Doppler broadening. This may be seen if one examines the efficiency of the optical pumping which is given by the following coefficient

$$
\eta \sim \frac{\Omega^{2} \gamma_{N} t}{(\Delta+\vec{k} \cdot \vec{v})^{2}+\Gamma^{2}}
$$

where $t$ is the time of interaction of the radiation with the atom, $\Delta$ is the detuning of the resonance, $\Gamma$ is the sum of homogeneous and non-homogeneous broadenings, $\gamma_{N}$ is the spontaneous decay of the considered transition, $\Omega$ is the Rabi frequency of the laser radiation which direction of propagation is $\vec{k}$ and $\vec{v}$ is the atomic velocity.

An obvious way to increase the coefficient (1) is to be as close as possible to the resonance $(\Delta \sim 0)$ and to perform a spectrum such that the experimental condition $\vec{k} \cdot \vec{v}=0$ is fulfilled. NC is an elegant and powerful device that realizes this condition: for the atoms flying perpendicularly to the laser beam the interaction time is $t_{\perp}=\frac{D}{v}$, where $D$ is the diameter of the laser beam, while for the atoms flying parallel to the laser beam, the interaction time is $t_{/ /}=\frac{L}{v}$. Thus, with a laser beam of diameter $D \sim 1 \mathrm{~mm}$ and distance between the walls of the $\mathrm{NC} L \sim 10^{2}-10^{3} \mathrm{~nm}, t_{\perp}$ is 3 to 4 orders higher than $t_{/ /}$. Moreover the atoms which fly perpendicularly to the laser beam verify the condition $\vec{k} \cdot \vec{v}=0$ and expression (1) takes the maximum value at $\Delta=0$, the VSOP peaks are exactly at the atomic transition frequencies. 
To demonstrate briefly the efficiency of the use of NCs to reduce the Doppler broadening, we show on Fig. 1 and 2 (respectively) the spectra for ordinary cell and $\mathrm{NC}$ (respectively) for the transition of ${ }^{87} \mathrm{Rb}$ $F_{g}=1 \rightarrow F_{e}=0,1,2$ and for the transition of ${ }^{87} \mathrm{Rb} F_{g}=1 \rightarrow F_{e}=1,2 ;{ }^{85} \mathrm{Rb} \quad F_{g}=2 \rightarrow F_{e}=2,3$ (respectively).

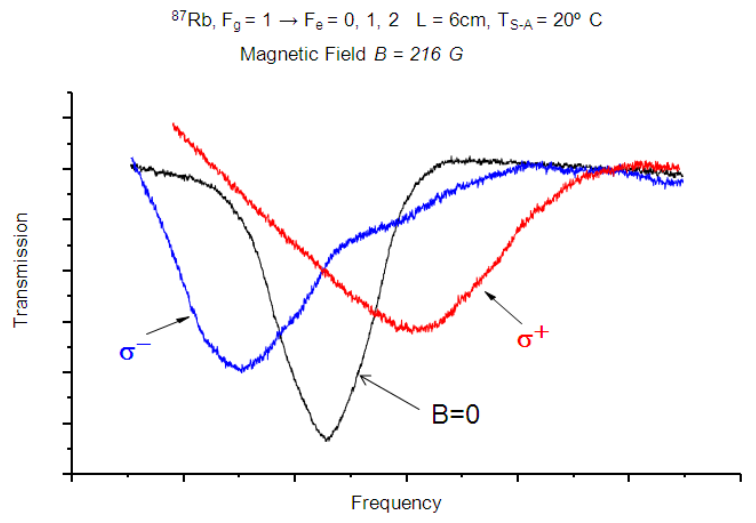

Fig. 1 Spectra of transmission in an ordinary cell for ${ }^{87} \mathrm{Rb}$ in the case $B=0$ and $B=200 \mathrm{G}$ for the radiations with polarisation $\sigma^{+}$ and $\sigma^{-}$.

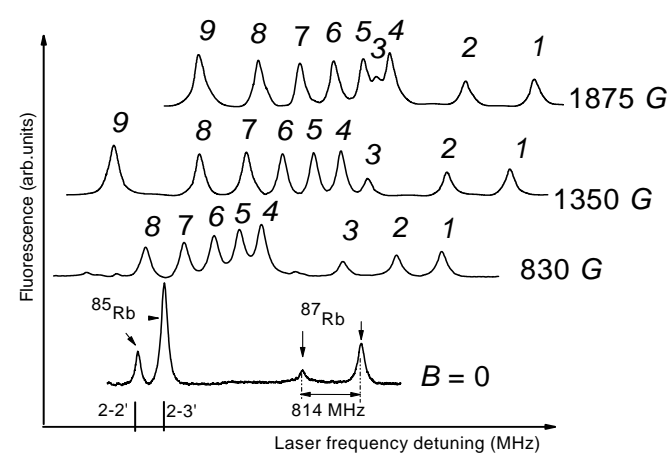

Fig. 2 Spectra of fluorescence in a $\mathrm{NC}$ for ${ }^{85} \mathrm{Rb}$ and ${ }^{87} \mathrm{Rb}$ for four values of $B$ from $0 \mathrm{G}$ to $1875 \mathrm{G}$.

In what follows, we will summarize the theoretical ingredients used to study the effect of an external magnetic field on the atomic transitions. Shifts of the Zeeman components and intensity of the corresponding transitions will be examined. Numerical applications to $\mathrm{Rb}$ and $\mathrm{Na}$ vapors confined in submicron thin vapor cell of thickness $L=\lambda / 2$ are compared with experimental results.

\section{EXPERIMENTAL PART}

Sketch of the experimental setup is presented in Fig.3a. The circularly polarized light of extended cavity diode laser (ECDL, $\lambda=780 \mathrm{~nm}, P_{L} \sim 30 \mathrm{~mW}, \gamma_{L}<1 \mathrm{MHz}$ ) resonant with ${ }^{87} \mathrm{Rb} \mathrm{D}_{2}$ transition frequency, after passing through Faraday isolator is directed onto the Rb NC 2 with the thickness of vapor column $L=\lambda / 2$, at an angle close to normal.

The needed temperature regime of the $\mathrm{NC}\left(\mathrm{T}_{\mathrm{SA}} \sim 110-120^{\circ} \mathrm{C}, \mathrm{T}_{\mathrm{W}} \sim 140-150{ }^{\circ} \mathrm{C}\right.$, corresponding to $N \sim 6 \times 10^{12}$ $-1.5 \times 10^{13}$ atoms $/ \mathrm{cm}^{3}$ ) is provided by a special oven with 3 openings: 2 inlets for laser beam passage and one orthogonal inlet for side fluorescence detection. This geometry allows simultaneous recording of fluorescence and transmission spectra. The fluorescence signal is detected by a photodiode 4 with an aperture of $1 \mathrm{~cm}^{2}$ which is placed at $90^{\circ}$ angle of the laser propagation direction. The photodiode signal is recorded by a digital four channel storage oscilloscope, Tektronix TDS 2014B.
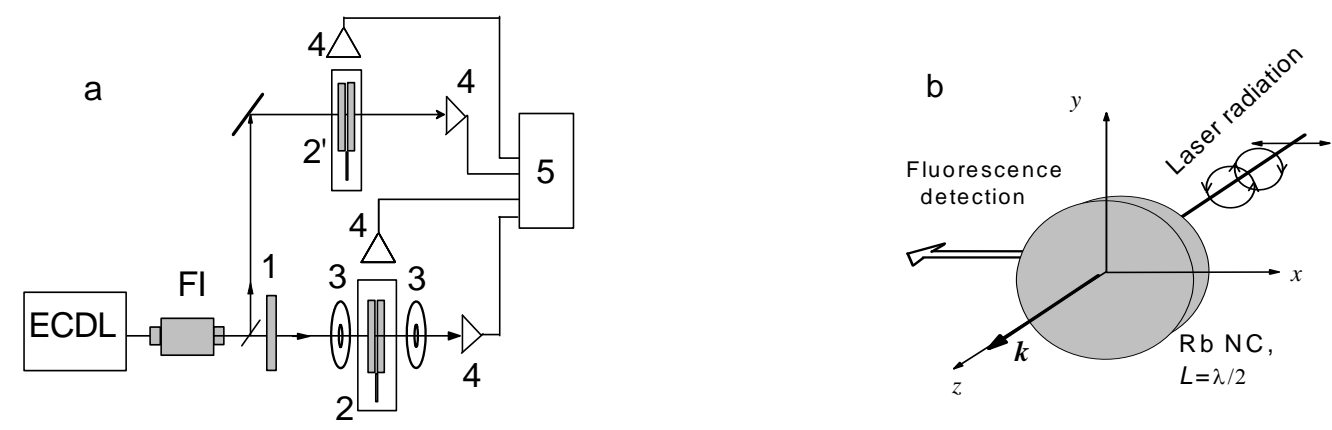

Fig. 3 a - Sketch of the experimental setup. FI - Faraday isolator, $1-\lambda / 4$ plate, 2 - STC and the oven, 2'- the auxiliary $\mathrm{NC}$ and the oven, 3 - RMs, 4 - photodetectors, 5 - digital storage oscilloscope; b - mutual orientations of laser excitation and detection of fluorescence emitted by a NC of $L=\lambda / 2$.

A Glan prism is used to purify initial linear radiation polarization of the laser radiation; to produce a circular polarization a $\lambda / 4$ plate 1 is utilized. The geometrical configuration of the experiment is shown in Fig. $3 \mathrm{~b}$. Magnetic field is directed along the laser radiation propagation direction $\boldsymbol{k}(\boldsymbol{B} / / \boldsymbol{k})$. 
With the help of a beam splitter, $50 \%$ of the pump beam is directed on the auxiliary 2 ' $\mathrm{NC}$ with $\mathrm{Rb}$ used as a frequency reference for $B=0$. In some cases the reference spectrum is transmission spectrum ${ }^{12}$ obtained with an auxiliary NC of thickness $L=\lambda$. The longitudinal magnetic field is applied to the NC by a system of Helmholtz coils (not shown in Fig.3a). The $B$-field strength is measured by a calibrated Hall gauge. Among the advantages of this setup is the possibility to apply strong magnetic field using widely available strong permanent magnets (PM). In spite of strong inhomogeneity of $B$-field, due to small thickness of the atomic vapor column inside NC $(L=390 \mathrm{~nm})$ the variation of $B$ inside the column is less than $0.1 \mathrm{G}$, i.e. by several orders less than the applied $B$ value.

\section{THEORETICAL BACKGROUND AND NUMERICAL CALCULATIONS}

The so-called Dirac equation ${ }^{8}$ for an electron to order $v^{2} / c^{2}$ is the starting point to study the spectra of an atom within an electromagnetic field describes by the potentials $\{\vec{A}, V\}$ :

$$
\left[\frac{1}{2 m}\left(\vec{p}+\frac{e}{c} \vec{A}\right)^{2}+\frac{e}{m c} \vec{S} \cdot \vec{\nabla} \wedge \vec{A}-\frac{p^{4}}{8 m^{3} c^{2}}-\frac{e \hbar}{8 m^{2} c^{2}} \Delta V-\frac{e}{2 m^{2} c^{2}} \vec{S} \cdot(\vec{\nabla} V \wedge \vec{p})-e V\right] \Psi=E \Psi
$$

with $\vec{S}=\frac{\hbar}{2} \vec{\sigma}$ where $\vec{\sigma}=\left(\sigma_{x}, \sigma_{y}, \sigma_{z}\right), \sigma_{i}(i=x, y, z)$ are the matrices of Pauli. For a potential $V=V(r)=\frac{Z e}{r}$, the term $-\frac{e}{2 m^{2} c^{2}} \vec{S} \cdot(\vec{\nabla} V \wedge \vec{p})$ describing the spin-orbit interaction is rewritten on a more compact form $H_{S O}=\xi(r) \vec{L} . \vec{S}$. It is worth to notice that this term leads naturally to calculate the Hamiltonian matrix in the basis $\left|n, L, J, F, m_{F}\right\rangle \equiv\left|F, m_{F}\right\rangle$ with $\vec{F}=\vec{I}+\vec{J}$ and $\vec{J}=\vec{L}+\vec{S}$. Thus for the Zeeman interaction, defined by $H_{Z}=-\vec{\mu}_{L} \cdot \vec{B}-\vec{\mu}_{S} \cdot \vec{B}-\vec{\mu}_{I} \cdot \vec{B}$ with $\vec{\mu}_{L}=-\frac{\beta}{\hbar} \vec{L}$ the magnetic orbital momentum, $\vec{\mu}_{S}=-\frac{2 \beta}{\hbar} \vec{S}$ the magnetic moment of spin $\vec{S}$ and $\vec{\mu}_{I}=-\frac{g_{I} \beta}{\hbar} \vec{I}$ the magnetic moment of the nuclear spin $\vec{I}$ where $\beta=\frac{e \hbar}{2 m c}$ is the Bohr magneton, the basis $\left|F, m_{F}\right\rangle$ can be chosen as a good basis to the first order if the condition $E_{Z}<<E_{S O}$ is fulfilled. For the magnetic field that we apply to the nano-cells, this condition is respected as the magnetic field will vary from 0 to $10^{4} \mathrm{G}$. One should note that all the previous discussion is valid for a magnetic field $\vec{B}$ supposed uniform $\left(\vec{A}=\frac{1}{2}(\vec{B} \wedge \vec{r})\right)$, which is what we have supposed in the development of the Zeeman interaction. Although interaction of atoms with laser radiation field in such cells is strictly anisotropic, however due to nanometric longitudinal size of the NCs, one may consider this hypothesis of uniformity as valid. This assumption is conformed by numerical calculation for inhomogeneity of $B$-field $150 \mathrm{G} / \mathrm{mm}$.

The Hamiltonian of interaction $H_{Z}=-\vec{\mu}_{L} \cdot \vec{B}-\vec{\mu}_{S} \cdot \vec{B}-\vec{\mu}_{I} \cdot \vec{B}$ may be expanded using different forms ${ }^{10-14}$, we chose a formulation ${ }^{10}$ which leads to

$$
H_{Z}=\left(-\frac{\mu_{B}}{\hbar}\right) \vec{B} \cdot\left(\vec{L}+g_{S} \vec{S}+g_{I} \vec{I}\right)
$$

where $g_{S}, g_{I}$ are respectively the spin and the nuclear Landé factor.

We adopt a matrix representation in the coupled basis, that is, the basis of the unperturbated atomic state vectors $\left|(n=3), L, J, F, m_{F}\right\rangle \equiv\left|F, m_{F}\right\rangle$ to evaluate the matrix elements of the Hamiltonian (3). In this basis, the diagonal matrix elements are given by

$$
\left\langle F, m_{F}|H| F, m_{F}\right\rangle=E_{0}(F)+\mu_{B} g_{F} m_{F} B_{Z}
$$

where $E_{0}(F)$ is the initial energy ${ }^{15}$ of the sublevel $\left|F, m_{F}\right\rangle$ and $g_{F}$ is the effective Landé factor ${ }^{15}$. 
a

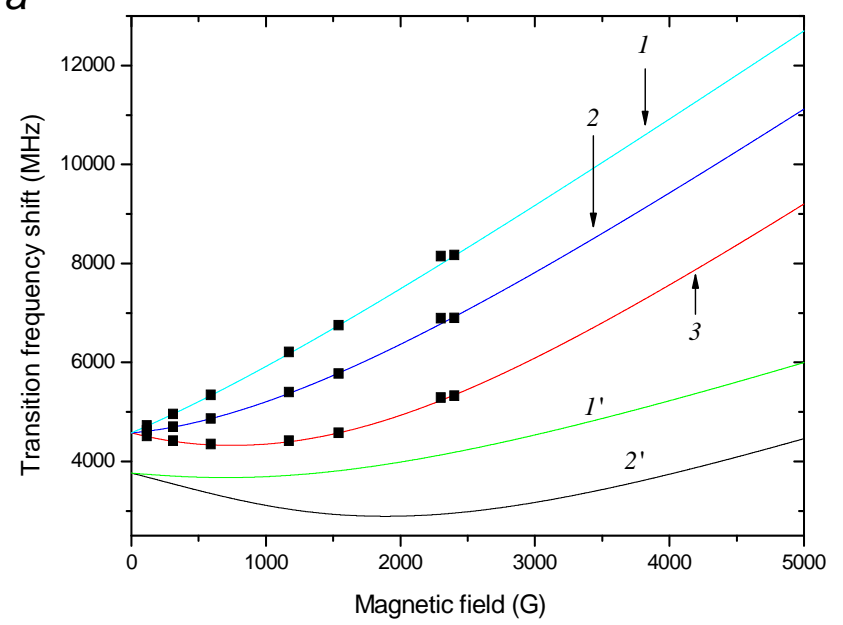

Fig. 4a Transition frequency shift versus magnetic field for ${ }^{87} \mathrm{Rb}, \mathrm{D}_{1}$ line

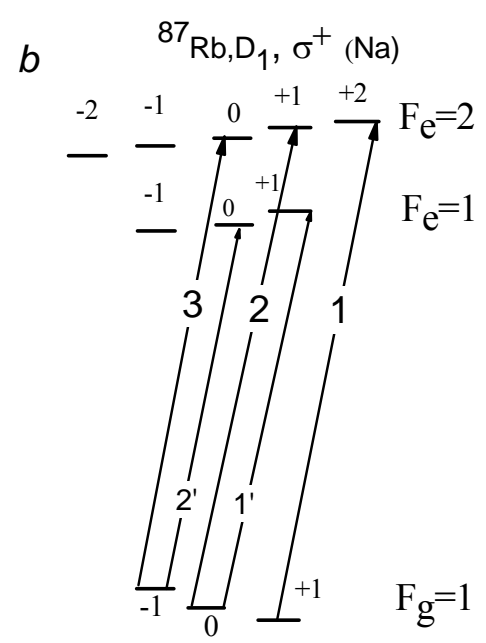

Fig. 4b Diagram of the labels 1, 2, 3 and $1^{\prime}, 2^{\prime}$

$$
\begin{aligned}
\left\langle F-1, m_{F}|H| F, m_{F}\right\rangle & =\left\langle F, m_{F}|H| F-1, m_{F}\right\rangle=-\frac{\mu_{B} B_{Z}}{2}\left(g_{J}-g_{I}\right) \\
\times & \left(\frac{\left[(J+I+1)^{2}-F^{2}\right]\left[F^{2}-(J-I)^{2}\right]}{F}\right)^{1 / 2}\left(\frac{F^{2}-m_{F}^{2}}{F(2 F+1)(2 F-1)}\right)^{1 / 2}
\end{aligned}
$$

The Hamiltonian matrix is then block diagonal where each block corresponds to a given value of $m_{F}$. The diagonalization of the Hamiltonian matrix allows one to find the eigenvectors and the eigenvalues, that is to determine the eigenvalues corresponding to the energies of Zeeman sublevels and the new states vectors which can be expressed in terms of the initial unperturbed atomic state vectors,

$$
\left|\Psi\left(F_{g}^{\prime}, m_{F_{g}}\right)\right\rangle=\sum_{F_{g}=3 / 2-J_{g}}^{F_{g}=3 / 2+J_{g}} \alpha_{F_{g}^{\prime} F_{g}}^{g}\left(B_{z}, m_{F_{g}}\right)\left|F_{g}, m_{F_{g}}\right\rangle \text { and }\left|\Psi\left(F_{e}^{\prime}, m_{F_{e}}\right)\right\rangle=\sum_{F_{g}=3 / 2-J_{g}}^{F_{g}=3 / 2+J_{g}} \alpha_{F_{e}^{\prime} F_{e}}^{e}\left(B_{z}, m_{F_{e}}\right)\left|F_{e}, m_{F_{e}}\right\rangle
$$

The state vectors $\left|F_{e}, m_{e}\right\rangle$ and $\left|F_{g}, m_{g}\right\rangle$ are the unperturbated state vectors, respectively, for the excited and the ground states. The coefficients $\alpha_{F_{e}^{\prime} F_{e}}^{e}\left(B_{z}, m_{F_{e}}\right)$ and $\alpha_{F_{g}^{\prime} F_{g}}^{g}\left(B_{z}, m_{F_{g}}\right)$ are mixing coefficients, respectively, for the excited and the ground states; they depend on the field strength and magnetic quantum numbers $m_{e}$ or $m_{g}$.

In figure $4 \mathrm{a}$ we present the energy levels obtained by the Hamiltonian matrix diagonalization for $D_{1}$ line of ${ }^{87} \mathrm{Rb}$ atom. Figure $4 \mathrm{~b}$ simply gives the definition of the notation used to label the transitions in Fig. $4 \mathrm{a}$.

The transition frequency shift values are obtained on a quasi continuous variation of the $B$ field intensity as we diagonalize 50000 matrices. From low $B$ field intensity to high $B$ field variation, this diagonalization procedure allows us to follow exactly the eigenvalues by analyzing precisely the jump of the eigenvectors using a standard derivative analysis method. One should note that Fig. 4a shows the observed frequency shift, measured experimentally (which are obtained from Fig.2) while simultaneously on the same curves have been superposed the calculated ones. As previously written, the hypothesis of locally homogeneous and uniform magnetic field is verified.

The probability of a transition, induced by the interaction of the atomic electric dipole and the oscillating laser electric field is proportional to the spontaneous emission rate of the associated transition $A_{e g}$, that is, to the square of the transfer coefficients modified by the presence of the magnetic field 


$$
\frac{8 \pi^{2}}{3 \varepsilon_{0} \hbar \lambda_{e g}^{3}}\left|\left\langle e\left|D_{q}\right| g\right\rangle\right|^{2}=A_{e g} \propto a^{2}\left[\Psi\left(F_{e}^{\prime}, m_{F_{e}}\right) ; \Psi\left(F_{g}^{\prime}, m_{F_{g}}\right) ; q\right]
$$

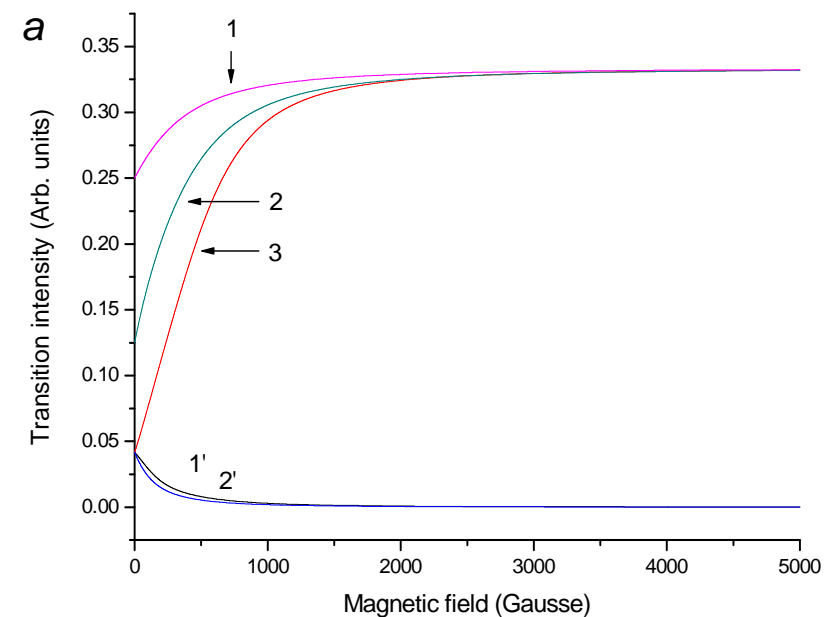

Fig.5a $D_{1}$ Line intensities for $\mathrm{Na}$, transitions $F_{g}=1 \rightarrow F_{e}=1,2$

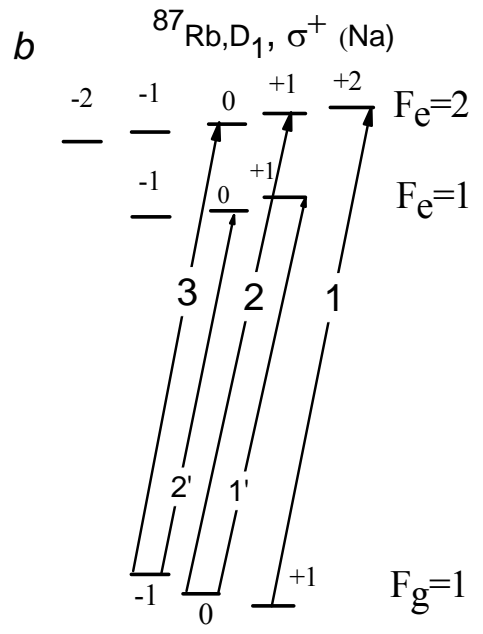

Fig.5b Diagram of the labels 1, 2, 3 and $1^{\prime}, 2^{\prime}$

where $D_{q} \quad(q=-1,0,1)$ denote the standard components of the electric dipole moment $\vec{D} \cdot \vec{e}=\sum_{q} D_{q} e_{q}$.

In eq. (7), the transfer coefficients are expressed as

$$
\left.a \mid \Psi\left(F_{e}^{\prime}, m_{F_{e}}\right) ; \Psi\left(F_{g}^{\prime}, m_{F_{g}}\right) ; q\right]=\sum_{F_{e}, F_{g}} \alpha_{F_{e}^{\prime} F_{e}}^{e}\left(B_{z}, m_{F_{e}}\right) a\left(F_{e}, m_{F_{e}} ; F_{g}, m_{F_{g}} ; q\right) \alpha_{F_{g}^{\prime} F_{g}}^{g}\left(B_{z}, m_{F_{g}}\right),
$$

where the unperturbated transfer coefficients have the following definition ${ }^{16}$

$$
a\left(F_{e}, m_{F_{e}} ; F_{g}, m_{F_{g}} ; q\right)=(-1)^{1+I+J_{e}+F_{e}+F_{g}-m_{F_{e}}} \sqrt{\left(2 J_{e}+1\right)\left(2 F_{e}+1\right)\left(2 F_{g}+1\right)}\left(\begin{array}{ccc}
F_{e} & 1 & F_{g} \\
-m_{F_{e}} & q & m_{F_{g}}
\end{array}\right)\left\{\begin{array}{lll}
F_{e} & 1 & F_{g} \\
J_{g} & I & J_{e}
\end{array}\right\}
$$

the parenthesis and curly brackets denote, respectively, the $3-j$ and $6-j$ symbols, $g$ and $e$ point respectively ground and excited states.

Figure 5a indicates clearly that the transition intensities versus the external magnetic field strength are strongly nonlinear for $B<1500 G$. For higher values of $B$, at least to $5000 G$, transitions $1,2,3$ tend asymptotically to the same limit while transitions $1^{\prime}, 2^{\prime}$ tend asymptotically to zero.

\section{CONCLUSION}

The $\lambda / 2$-Zeeman technique is shown to be a convenient and robust method for the study of individual transitions between the Zeeman sublevels of hyperfine levels in an external magnetic field. NC in external magnetic field allows exhibiting obviously the several Zeeman components due mainly to the suppression of the Doppler broadening. One of the goals of the construction of such an elegant tool was the possibility to remove this effect and it succeeds perfectly. As a result, the use of a NC as a nanomagnetometer seems a successful application of this device as it allows the measurement of extremely large gradient of magnetic field. Calculated and experimental results are in very good agreement.

\section{Acknowledgement}

The authors are grateful to A. Sarkisyan for his valuable participation in fabrication of the STC as well as to A. Papoyan for useful discussions. Research conducted in the scope of the International Associated Laboratory IRMAS. Armenian team thanks for a research grant PS 1868 from the Armenian National Science and Education Fund (ANSEF) based in New York, USA. 


\section{References}

[1] D. Sarkisyan, D. Bloch, A. Papoyan and M. Ducloy, "Sub-Doppler spectroscopy by sub-micron thin Cs vapour layer", Opt. Commun. 200, 201 - 208 (2001).

[2] G. Dutier, A. Yarovitski, S. Saltiel, A. Papoyan, D. Sarkisyan, D. Bloch and M. Ducloy, "Collapse and revival of a Dicke-type coherent narrowing in a sub-micron thick vapor cell transmission spectroscopy", Europhys. Lett. 63 (1), 35 - 41 (2003).

[3] D. Sarkisyan, T. Becker, A. Papoyan, P. Thoumany and H. Walther, "Sub-Doppler Fluorescence on atomic $\mathrm{D}_{2}$ line of Sub-Micron Rubidium Vapor Layer”, Appl. Phys. B 76, N6, pp. 625 - 631 (2003).

[4] D. Sarkisyan, T. Varzhapetyan, A. Sarkisyan, Yu. Malakyan, A. Papoyan, A. Lezama, D. Bloch and M. Ducloy, "Spectroscopy in an extremely thin vapor cell: Comparing the cell-length dependence in fluorescence and in absorption techniques", Phys. Rev. A 69, 065802 (4p.) (2004).

[5] A. Papoyan, D. Sarkisyan, K. Blush, M. Auzinsh, D. Bloch and M. Ducloy, "Magnetic field-induced mixing of hyperfine states of the Cs 6P level observed with a submicron vapor cell", Laser Physics 13, 1467 - 1477 (2003).

[6] D. Sarkisyan, A. Papoyan, T. Varzhapetyan, K. Blush and M. Auzinsh, "Hyperfine structure Zeeman effect on of an atomic D1 line of a sub-micron ${ }^{87}$ Rb vapor layer", Opt. and Spectrosc. 96, 328 - 334 (2004).

[7] D. Sarkisyan, A. Papoyan, T. Varzhapetyan, K. Blush and M. Auzinsh, "Fluorescence of rubidium in a submicrometer vapor cell: spectral resolution of atomic transitions between Zeeman sublevels in a moderate magnetic field", J. Opt. Soc. Am. B 22, 88 - 95 (2005).

[8] M. Weissbluth, Atoms and Molecules. Academic, New-York (1978) and references herein.

[9] A. Sargsyan, G. Hakhumyan, A. Papoyan, D. Sarkisyan, A. Atvars and M. Auzinsh, "A novel approach to quantitative spectroscopy of atoms in a magnetic field and applications based on an atomic vapor cell with $L=\lambda$ ”, Appl. Phys. Lett. 93, 021119 (3p.) (2008).

[10] P. Tremblay, A. Nichaud, M. Levesque, S. Theriault, M. Breton, J. Beaubien, and N. Cyr, “Absorption profiles of alkali-metal D lines in the presence of a static magnetic field," Phys. Rev. A 42, 2766 (1990).

[11] E. B. Aleksandrov, M. P. Chaika, and G. I. Khvostenko, Interference of Atomic States (Springer-Verlag, New York, 1993).

[12] V. Papoyan, D. H. Sarkisyan, K. Blush, M. Auzinsh, D. Bloch, and M. Ducloy, "Magnetic Field-Induced Mixing of the Cs $6^{2} P_{3 / 2}$ Level Observed with a Submicron Vapor Cell", Laser Physics 13, 1-11 (2003).

[13] B. W. Shore, The Theory of Coherent Atomic Excitation, Multilevel Atoms and Incoherence (Wiley Interscience, New York, 1990).

[14] D. Sarkisyan, A. Papoyan, T. Vazhapetyan, J. Alnis, K. Blush and M. Auzinsh, "Sub-Doppler spectroscopy of $\mathrm{Rb}$ atoms in a sub-micron vapour cell in the presence of a magnetic field", J. Opt. A: Pure Appl. Opt. 6, S142-S150 (2004).

[15] Daniel A. Steck, "Sodium D Line Data," available online at http://steck.us/alkalidata (revision 2.1.3, 26 August 2009).

[16] E. de Clercq, M. de Labachellerie, G. Avila, P. Cerez and M. Tetu, "Laser diode optically pumped caesium beam,” J. Phys. France 45, 239-247 (1984). 